\title{
A Review on Articles in the Current Issue
}

\author{
Hsuan-Hsiang Chen* \\ Department of Dermatology, National Taiwan University Hospital, 7, Chung-Shan South Road, Taipei, Taiwan
}

Received: 15 November, 2014; Accepted: 15 November, 2014; Published: 15 November, 2014

*Corresponding author: Hsuan-Hsiang Chen, MD, Department of Dermatology, National Taiwan University Hospital, 7, Chung-Shan South Road, Taipei, Taiwan, Tel.: +886-2-2356-2141; Fax: +886-2-2393-4177; E-mail: beauty101@gmail.com

It is a great pleasure to introduce a new type of dermatological journal here. Clinical Research in Dermatology: Open Access (CRDOA) is a new international journal aimed to provide interesting and intriguing research in dermatology through free online open access.In this inaugural issue, we have some interesting scientific findings from Turkey, Australia, America, Taiwan, and Brazil.

Cutaneous findings always provide an insight to the underlying systemic disease during in-patient dermatological consultation. Demir et al. [1] found most consultations were requested from Internal medicine and the patients over the age of 60 years. Drug eruption was the most frequent diagnosis, probably reflecting the in-patients taking lots of drugs when hospitalized. Over half of dermatologic diagnoses were incorrect by the nondermatologists, revealing the importance of dermatology branch in the hospital setting.

The evaluation tool in a residency program is always important during residents' training. Morrell et al. [2] compared two versions of the 360-degree evaluation tool and demonstrated the shorted format one (360-2) offers a better efficient and valid method for resident assessment, because the initial longer version (360-1) can cause evaluation fatigue.

The literature review by Lalji et al. [3] tried to assess the differences between actinic keratosis and squamous cell carcinoma (SCC). They found it is difficult to distinguish between these two conditions in Pubmed, Cochrane and Medline databases. They suggested early in situ SCC type AK1, AK2, and AK3 classification system may be considered.

Mango may cause either type I or type IV hypersensitivity. Chen et al. [4] tried various extracts from the pulp, peel-lining, and peel of two different mangos and showed the $10 \%$ ether extract can be used as a screening tool to evaluate the mango dermatitis. They also discussed the relationship between hypersensitivity to poison oak/ivy and mango dermatitis. They proposed the "alkyl" or "alkenyl' group of the resorcinols (from mango) and the catechols (from poison oak/ivy) may be recognized as a common epitope.

Finally, Sampaio et al. [5] reported an interesting case of cutaneous Leishmaniasis after a laser hair removal in a 30-yearold female. They reminded us the possibility of promastigote inoculation through local trauma.

\section{References}

1. Demir FT, Can B, Zindanci I, Turkoglu Z, Kavala M, Kocaturk E. Dermatology In-Patients in a University Teaching Hospital. Clin Res Dermatol Open Access. 2014;1(1):1-2. DOI: doi.org/10.15226/2378$1726 / 1 / 1 / 00101$

2. Dean S Morrell, Sam wu, Sasha N Jenkins, Elizabeth R Hoffman. Validity and Use of the 360-Degree Evaluation Tool in a Dermatology Residency Program. Clin Res Dermatol Open Access. 2014;1(1):1-4. DOI: doi.org/10.15226/2378-1726/1/1/00103

3. Lalji A, Khiroya N and Lalji M. Actinic Keratosis and Squamous Cell Carcinoma. Clin Res Dermatol Open Access. 2014;1(1):1-3. DOI: doi. org/10.15226/2378-1726/1/1/00102

4. Hsuan-Hsiang Chen and Chee-Ching Sun. Patch Testing with Various Mango (Mangifera indica) Extracts. Clin Res Dermatol Open Access. 2014;1(1):1-3. DOI: doi.org/10.15226/2378-1726/1/1/00104

5. Raimunda NR Sampaio, Marcela ST Mendes, Daniel G, Jefferson BP Ribeiro, Adriana OS Alfani, Beatriz D Lima, et al. Cutaneous Leishmaniasis Following Local Trauma: A Case Report. Clin Res Dermatol Open Access. 2014;1(1):1-3. DOI: doi.org/10.15226/23781726/1/1/00105. 